MOHAMMED BEKKA

PIERRE DE LA HARPE

Représentations d'un groupe faiblement équivalentes à la représentation régulière

Bulletin de la S. M. F., tome 122, no 3 (1994), p. 333-342

$<$ <ttp://www.numdam.org/item?id=BSMF_1994_122_3_333_0>

(C) Bulletin de la S. M. F., 1994, tous droits réservés.

L'accès aux archives de la revue «Bulletin de la S. M. F. » (http: //smf.emath.fr/Publications/Bulletin/Presentation.html) implique l'accord avec les conditions générales d'utilisation (http://www.numdam.org/ conditions). Toute utilisation commerciale ou impression systématique est constitutive d'une infraction pénale. Toute copie ou impression de ce fichier doit contenir la présente mention de copyright.

\title{
Numdam
}


Bull. Soc. math. France,

122, 1994, p. 333-342.

\section{REPRÉSENTATIONS D'UN GROUPE \\ FAIBLEMENT ÉQUIVALENTES À LA \\ REPRÉSENTATION RÉGULIÈRE}

PAR

Mohammed BEKKA et PIERRE DE LA HARPE (*)

RÉSUMÉ. - Soit $\Gamma$ un réseau dans un groupe de Lie réel connexe $G$ qui est simple, de centre réduit à $\{1\}$, et non compact. Soient $\pi$ une représentation unitaire de $G$ et $\left.\pi\right|_{\Gamma}$ sa restriction à $\Gamma$. Si $\pi$ n'est pas triviale, on montre que $\left.\pi\right|_{\Gamma}$ contient faiblement la représentation régulière $\lambda_{\Gamma}$ de $\Gamma$. Lorsque $\pi$ est dans la série principale ou dans la série discrète de $G$, ceci implique que la représentation $\pi_{\mid \Gamma}$ est faiblement équivalente à $\lambda_{\Gamma}$. Dans le cas où $G=\operatorname{PSL}_{2}(\mathbb{R})$ et lorsque $\pi$ est dans la série complémentaire, on obtient aussi des résultats sur $\left.\pi\right|_{\Gamma}$.

Abstract. - Let $\Gamma$ be a lattice in a connected real Lie group $G$ which is simple, non compact and with centre reduced to $\{1\}$. Let $\pi$ be a unitary representation of $G$ and let $\pi_{\mid \Gamma}$ be its restricition to $\Gamma$. If $\pi$ is not trivial, we show that $\pi_{\mid \Gamma}$ weakly contains the regular representation $\lambda_{\Gamma}$ of $\Gamma$. When $\pi$ is in the principal series or the discrete series of $G$, this implies that $\pi_{\left.\right|_{\Gamma}}$ and $\lambda_{\Gamma}$ are weakly equivalent. In the case $G=\mathrm{PSL}_{2}(\mathbb{R})$ and when $\pi$ is in the complementary series, we obtain also results on $\pi_{\mid \Gamma}$.

\section{Introduction}

Soit $G$ un groupe localement compact. Les représentations de $G$ considérées ici sont les représentations unitaires continues de $G$ dans des espaces de Hilbert complexes non nuls. Étant donné une telle représentation $\pi$ dans $\mathcal{H}_{\pi}$, les fonctions de type positif associées à $\pi$

(*) Texte reçu le 28 septembre 1992, révisé le 26 novembre 1992.

M. BEKKA, Département de Mathématiques, Université de Lausanne, 1015 Lausanne, Suisse et Mathematisches Institut, Technische Universität Munchen, Arcisstr. 21, D-8000, München 2, Allemagne.

P. DE LA HARPE, Section de Mathématiques, Université de Genève, C.P. 240, $\mathrm{CH}-1211$, Genève 24, Suisse.

Mots clés : représentations unitaires, contenance faible, réseaux, groupes de Lie semi-simples.

Classification AMS : 22 D 10, 22 E 40.

BULlETIN DE LA SOCIÉtÉ MATHÉMATIQUE DE FRANCE 0037-9484/1994/333/\$5.00

(C) Société mathématique de France 
sont les fonctions $G \rightarrow \mathbb{C}$ de la forme $g \mapsto\langle\xi \mid \pi(g) \xi\rangle$ où $\xi$ est un vecteur de $\mathcal{H}_{\pi}$. Soient $\pi, \pi^{\prime}$ deux représentations de $G$; on dit que $\pi$ est faiblement contenue dans $\pi^{\prime}$ et on écrit $\pi \prec \pi^{\prime}$ si les fonctions de type positif associées à $\pi$ sont approchables par des sommes de fonctions de type positif associées à $\pi^{\prime}$. («Approchables》 veut dire ici «limites uniformes sur tout compact de $G »$.) On dit que $\pi$ et $\pi^{\prime}$ sont faiblement équivalentes et on écrit $\pi \sim \pi^{\prime}$ si $\pi \prec \pi^{\prime}$ et $\pi^{\prime} \prec \pi$. Pour une introduction à ces notions, voir par exemple le livre de Dixmier [DC*].

On note $L^{2}(G)$ l'espace des fonctions complexes de carré sommable sur $G$ pour une mesure de Haar invariante à gauche; on note $\lambda_{G}$ la représentation régulière gauche de $G$ dans $L^{2}(G)$. Les questions qui nous intéressent ici concernent un sous-groupe discret $\Gamma$ d'un groupe de Lie $G$. Toute représentation $\pi$ de $G$ fournit par restriction une représentation $\pi_{\mid \Gamma}$ de $\Gamma$ (agissant dans le même espace que $\pi$ ). Nous cherchons à comprendre quand $\pi_{\mid \Gamma}$ contient faiblement la représentation régulière $\lambda_{\Gamma}$ de $\Gamma$, et plus généralement quelles sont les propriétés de l'application $\pi \mapsto \pi_{\mid \Gamma}$ du point de vue de la contenance faible.

Soit $C^{*}(G)$ la $C^{*}$-algèbre de $G$. Soit $\pi$ une représentation de $G$ dans un espace $\mathcal{H}_{\pi}$ et soit $\mathcal{L}\left(\mathcal{H}_{\pi}\right)$ la $C^{*}$-algèbre des opérateurs bornés sur $\mathcal{H}_{\pi}$. Alors $\pi$ induit un morphisme de $C^{*}$-algèbres $C^{*}(G) \rightarrow \mathcal{L}\left(\mathcal{H}_{\pi}\right)$ encore noté $\pi$, avec noyau et image respectivement notés $C^{*} \operatorname{Ker}(\pi)$ et $C_{\pi}^{*}(G)$. Si $\pi=\lambda_{G}$, la $C^{*}$-algèbre $C_{\lambda_{G}}^{*}(G)$ est la $C^{*}$-algèbre réduite $C_{\text {red }}^{*}(G)$ de $G$. Si $\pi$ et $\pi^{\prime}$ sont deux représentations de $G$, rappelons qu'on a $\pi \prec \pi^{\prime}$ si et seulement si $C^{*} \operatorname{Ker}(\pi) \supset C^{*} \operatorname{Ker}\left(\pi^{\prime}\right)$. (Voir dans [DC*] les numéros 3.4.4 et 18.1.4.)

Les notations et considérations précédentes valent aussi pour un sousgroupe discret $\Gamma$ de $G$. Supposons de plus $\Gamma$ tel que $C_{\text {red }}^{*}(\Gamma)$ soit une $\mathrm{C}^{*}$-algèbre simple (pour des exemples, voir entre autres [BGH], [Har], $[\mathrm{HoR}])$; pour toute représentation $\rho$ de $\Gamma$, on a dans ce cas :

$$
\rho \prec \lambda_{\Gamma} \Longleftrightarrow \rho \sim \lambda_{\Gamma} \text {. }
$$

Toutefois, pour beaucoup des groupes $\Gamma$ qui apparaissent ci-dessous, on ignore si les $\mathrm{C}^{*}$-algèbres réduites correspondantes sont simples.

\section{Résultats}

Soit $G$ un groupe de Lie réel connexe qui est simple, non compact, et de centre réduit à $\{1\}$. Soit $B=M A N$ un sous-groupe parabolique minimal de $G$; rappelons que la série principale de $G$ est la famille des représentations de la forme $\operatorname{Ind}_{B}^{G}(\rho)$, où $\rho$ est une représentation de $B$ dont la restriction à $M$ (resp. $A, N$ ) est irréductible (resp. un caractère unitaire de $A$, la représentation triviale de $N$ ); pour tout ceci, voir par

TOME $122-1994-\mathrm{N}^{\circ} 3$ 
exemple [Wal]. Rappelons aussi que la plupart de ces représentations (mais pas toutes) sont irréductibles [Wal, th. 8.3.12]. Convenons qu'une représentation $\pi$ est triviale si $\pi(g) \xi=\xi$ pour tous $g \in G$ et $\xi \in \mathcal{H}_{\pi}$; on désigne par $1_{G}$ la représentation triviale de $G$ de dimension 1 . Notons que toute représentation non triviale de $G$ est fidèle, car $G$ est simple. Rappelons que $G$ est un groupe linéaire (via sa représentation adjointe) et que la topologie de Zariski est donc bien définie sur $G$ [Zim, prop. 3.1.6].

THÉORÈmE 1. - On se donne un groupe simple $G$ comme ci-dessus et un sous-groupe discret $\Gamma$ de $G$ dense pour la topologie de Zariski, par exemple un réseau de $G$. Soit $\pi$ une représentation de $G$.

(i) Si $\pi$ n'est pas triviale, alors $\lambda_{\Gamma} \prec \pi_{\mid \Gamma}$.

(ii) Si $\pi \prec \lambda_{G}$, par exemple si $\pi$ est dans la série principale, alors $\lambda_{\Gamma} \sim \pi_{\mid \Gamma}$.

Dans la situation du ThÉorÈme 1 et lorsque $\Gamma$ est un réseau de $G$, rappelons le résultat suivant de CowLING et Steger [CoS] :

(iii) Si $\pi$ est irréductible et n'est pas de carré intégrable, alors $\pi_{\mid \Gamma}$ est irréductible; de plus, si $\pi$ et $\pi^{\prime}$ sont deux représentations irréductibles inéquivalentes de $G$ qui ne sont pas de carré intégrable, alors $\pi_{\mid \Gamma}$ et $\pi^{\prime}{ }_{\mid \Gamma}$ ne sont pas équivalentes.

On sait par ailleurs qu'on a [GHJ, § 3.3.c] :

(iv) si $\pi$ est irréductible dans la série discrète de $G$, alors $\pi_{\mid \Gamma}$ est quasi-équivalente à $\lambda_{\Gamma}$ (au sens de [DC*, $\mathrm{n}^{\mathrm{o}}$ 5.3.1]).

Considérons le cas particulier de $G=\mathrm{PSL}_{2}(\mathbb{R})$ et d'une représentation irréductible $\pi$ de $G$. Si $\pi$ est dans la série principale ou dans la série discrète, voir (ii) et (iv) ci-dessus. Les autres représentations irréductibles de $G$ constituent la série complémentaire $\left(\pi_{t}\right)_{0<t<1}$, paramétrée ici comme dans [Lan]. Nous notons $\pi_{t, \Gamma}$ la restriction de $\pi_{t}$ à un sous-groupe discret $\Gamma \operatorname{de} G$.

ThÉORÈme 2. - Soient $\Gamma$ un réseau de $G=\mathrm{PSL}_{2}(\mathbb{R})$ et $\pi_{s}, \pi_{t}$ deux représentations de la série complémentaire de $G$ telles que $s<t$. Alors :

(v) $\lambda_{\Gamma} \prec \pi_{t, \Gamma}$ et $\lambda_{\Gamma} \nsim \pi_{t, \Gamma}$,

(vi) $\pi_{t, \Gamma} \nprec \pi_{s, \Gamma}$.

Dans la situation du THÉorème 2 , on sait que $C_{\text {red }}^{*}(\Gamma)$ est simple [Har], et il résulte de (v) qu'on a des morphismes surjectifs :

$$
\phi_{t}: C_{\pi_{t, \Gamma}}^{*}(\Gamma) \longrightarrow C_{\mathrm{red}}^{*}(\Gamma) .
$$

Il serait intéressant d'étudier les noyaux de ces morphismes. (Au niveau du groupe de Lie, rappelons que $G$ est liminaire [DC*, th. 15.5.6]; par 
suite $C_{\pi}^{*}(G)$ est l'idéal des opérateurs compacts dans $\mathcal{L}\left(\mathcal{H}_{\pi}\right)$ pour toute représentation irréductible $\pi$ de $G$, de sorte que $C_{\pi}^{*}(G)$ est simple pour tout $\pi \in \widehat{G})$.

Dans le cas où $\Gamma=\mathrm{PSL}_{2}(\mathbb{Z})$, nous pouvons préciser l'assertion (vi) ci-dessus. Auparavant, rappelons que le dual unitaire $\widehat{G}$ d'un groupe localement compact $G$ est muni de la topologie de Fell, pour laquelle l'adhérence d'une partie $A \subset \widehat{G}$ est définie par

$$
\bar{A}=\left\{\pi \in \widehat{G}: \pi \prec \bigoplus_{\rho \in A} \rho\right\}
$$

(voir [DC*, chap. 18]). Pour $G=\mathrm{PSL}_{2}(\mathbb{R})$, il est bien connu que l'application

$$
] 0,1\left[\longrightarrow \widehat{G}, \quad t \mapsto \pi_{t},\right.
$$

paramétrisant la série complémentaire, est un homéomorphisme sur son image (voir [Del, p. 306] et [EhM, th. 1 et 3]). Si $\Gamma$ est un réseau de $G$, on a en vertu de l'assertion (iii) ci-dessus une application

$$
] 0,1\left[\longrightarrow \widehat{\Gamma}, \quad t \mapsto \pi_{t, \Gamma}\right.
$$

qui est injective.

ThÉORÈmE 3. - On considère le groupe modulaire $\Gamma=\mathrm{PSL}_{2}(\mathbb{Z})$ comme un réseau dans $G=\mathrm{PSL}_{2}(\mathbb{R})$. Pour tous $\left.s, t \in\right] 0,1[$ tels que $s \neq t$, on $a$ :

(vii) $\pi_{s, \Gamma} \nprec \pi_{t, \Gamma}$.

Plus précisément, l'application

$$
] 0,1\left[\longrightarrow \widehat{\Gamma}, \quad t \mapsto \pi_{t, \Gamma}\right.
$$

est un homéomorphisme sur son image.

La preuve de ce théorème utilise des résultats de Selberg sur la représentation quasi-régulière de $\mathrm{PSL}_{2}(\mathbb{R})$ dans $\mathrm{PSL}_{2}(\mathbb{R}) / \mathrm{PSL}_{2}(\mathbb{Z})$. Des résultats analogues sont conjecturés pour tous les sous-groupes de congruence de $\mathrm{PSL}_{2}(\mathbb{Z})$. Si cette «conjecture de Selberg» est vraie, le THÉORÈme 3 et sa preuve valent aussi pour ces sous-groupes.

TOME $122-1994-\mathrm{N}^{\circ} 3$ 


\section{Preuves}

Convenons qu'un groupe $\Gamma$ est fortement $c c i$ si, pour toute partie finie $F$ de $\Gamma$ disjointe de $\{1\}$, il existe une suite infinie $\left(\gamma_{j}\right)_{j \geq 1}$ d'éléments de $\Gamma$ telle que, pour tout $x \in F$, les éléments $\gamma_{j}^{-1} x \gamma_{j}$ sont distincts deux à deux. Cette propriété renforce manifestement celle d'être à «classes de conjugaison infinies », qui est classique [MvN, lemme 5.3.4].

Soit $G$ un groupe localement compact. Une représentation $\pi$ de $G$ est dite de classe $\left(\mathcal{C}_{0}\right)$ s'il existe un vecteur unité $\xi \in \mathcal{H}_{\pi}$ tel que la fonction de type positif $g \mapsto\langle\xi \mid \pi(g) \xi\rangle$ tende vers 0 à l'infini de $G$.

Proposition 1. - Soit $\Gamma$ un groupe fortement cci et soit $\pi$ une représentation de $\Gamma$ de classe $\left(\mathcal{C}_{0}\right)$. On a $\lambda_{\Gamma} \prec \pi$.

Preuve. - Soit $\delta: \Gamma \rightarrow \mathbb{C}$ la fonction définie par $\delta(\gamma)=\left\langle\xi_{1} \mid \lambda_{\Gamma}(\gamma) \xi_{1}\right\rangle$ où $\xi_{1} \in \ell^{2}(\Gamma)$ est la fonction caractéristique de $\{1\}$; on a donc $\delta(1)=1$ et $\delta(\gamma)=0$ si $\gamma \neq 1$. Vu [DC*, prop. 18.1.4], il suffit de vérifier que $\delta$ est approchable par des fonctions de type positif associées à $\pi$.

Soit $F$ une partie finie de $\Gamma$ disjointe de $\{1\}$. Soit $\left(\gamma_{j}\right)_{j \geq 1}$ une suite comme dans la définition de «fortement cci». Par hypothèse sur $\pi$, on peut choisir un vecteur unité $\xi_{0} \in \mathcal{H}_{\pi}$ tel que

$$
\lim _{j \rightarrow \infty}\left\langle\xi_{0} \mid \pi\left(\gamma_{j}^{-1} x \gamma_{j}\right) \xi_{0}\right\rangle=0 \quad \text { pour tout } x \in F
$$

car $\gamma_{j}^{-1} x \gamma_{j} \neq \gamma_{k}^{-1} x \gamma_{k}$ si $j \neq k$. Si on définit $\phi_{F, j}: \Gamma \rightarrow \mathbb{C}$ par

$$
\phi_{F, j}(\gamma)=\left\langle\pi\left(\gamma_{j}\right) \xi_{0} \mid \pi(\gamma) \pi\left(\gamma_{j}\right) \xi_{0}\right\rangle
$$

on a donc bien $\lim _{j \rightarrow \infty} \phi_{F, j}(x)=\delta(x)$ pour tout $x \in F \cup\{1\}$.

Proposition 2. - Soit $G$ un groupe de Lie réel connexe qui est semi-simple, de centre réduit à $\{1\}$, et sans facteur compact. Soit $\Gamma$ un sous-groupe discret de $G$ dense pour la topologie de Zariski. Alors $\Gamma$ est fortement cci.

Preuve. - Soit $F$ une partie finie de $\Gamma$ disjointe de $\{1\}$. Il s'agit de montrer qu'il existe une suite infinie $\left(\gamma_{j}\right)_{j \geq 1}$ avec les propriétés requises. On pose $\gamma_{1}=1$ et on procède par récurrence. Supposons qu'il existe une suite $\left(\gamma_{j}\right)_{1 \leq j \leq k}$ telle que, pour tout $x \in F$, les éléments $\gamma_{j}^{-1} x \gamma_{j}$ sont distincts deux à deux. Pour chaque $j \in\{1, \ldots, k\}$ et chaque $x \in F$, le fermé de Zariski

$$
Z_{G}(x) \gamma_{j}=\left\{y \in G: y^{-1} x y=\gamma_{j}^{-1} x \gamma_{j}\right\}
$$

BULLETIN DE LA SOCIÉTÉ MATHÉMATIQUE DE FRANCE 
est distinct de $G$ (la notation $Z_{G} \gamma_{j}$ indique le translaté par $\gamma_{j}$ d'un centralisateur). Comme la réunion d'un nombre fini de fermés de Zariski distincts de $G$ est distincte de $G$, il suffit de choisir $\gamma_{k+1} \in \Gamma$ tel que

$$
\gamma_{k+1} \notin \bigcup_{1 \leq j \leq k} \bigcup_{x \in F} Z_{G}(x) \gamma_{j}
$$

pour avoir les propriétés désirées.

(Notons que la proposition et sa preuve valent pour un groupe de Lie algébrique réel de centre réduit à $\{1\}$.)

Nous ignorons s'il existe des groupes à classes de conjugaison (autres que $\{1\})$ infinies qui ne sont pas fortement cci.

Lemme. - Soient $G$ un groupe localement compact, $H$ un sous-groupe fermé de $G$ et $\pi$ une représentation de $G$.

(i) Si $\pi \prec \lambda_{G}$, alors $\pi_{\mid H} \prec \lambda_{H}$.

(ii) On suppose de plus que la représentation triviale $1_{G}$ de $G$ est faiblement contenue dans la représentation $\operatorname{Ind}_{H}^{G}\left(1_{H}\right) . S i \pi_{\mid H} \prec \lambda_{H}$, alors $\pi \prec \lambda_{G}$.

Preuve. - L'assertion (i) résulte de ce que $\pi \prec \lambda_{G}$ si et seulement si les fonctions de type positif associées à $\pi$ sont approchables par des fonctions de type positif à supports compacts [DC*, prop. 18.3.5], et de ce que cette dernière condition passe évidemment de $\pi$ à sa restriction $\pi_{\mid H}$.

Avant de montrer (ii), rappelons que $\operatorname{Ind}_{H}^{G}\left(\pi_{\mid H}\right)=\pi \otimes \operatorname{Ind}_{H}^{G}\left(1_{H}\right)$; voir par exemple le théorème 6 au paragraphe VI.11 de [Gaa]. Supposons alors que $\pi_{\mid H} \prec \lambda_{H}$. En induisant de $H$ à $G$, on obtient par «continuité de l'induction $\gg[$ Fel, th. 4.1] :

$$
\pi \otimes \operatorname{Ind}_{H}^{G}\left(1_{H}\right) \prec \operatorname{Ind}_{H}^{G}\left(\lambda_{H}\right)=\lambda_{G}
$$

Si $1_{G} \prec \operatorname{Ind}_{H}^{G}\left(1_{H}\right)$, on a donc bien $\pi=\pi \otimes 1_{G} \prec \lambda_{G}$.

Remarque. - La condition $1_{G} \prec \operatorname{Ind}_{H}^{G}\left(1_{H}\right)$ de (ii) signifie que $G / H$ est un espace moyennable au sens d'EYMARD [Eym]. Elle est évidemment satisfaite si $H$ est un réseau dans $G$, auquel cas $1_{G}$ est une sousreprésentation de $\operatorname{Ind}_{H}^{G}\left(1_{H}\right)$.

Preuve du théorème 1. - Notons d'abord que la Proposition 2 s'applique à un réseau de $G$ grâce au théorème de densité de Borel; voir [Bor] ou [Zim, th. 3.2.5]. Rappelons que toute représentation non triviale de $G$ est de classe $\left(\mathcal{C}_{0}\right)$ en vertu du théorème 5.2 de [HoM].

TOME $122-1994-\mathrm{N}^{\circ} 3$ 
L'assertion (i) résulte donc de ce que la propriété $\left(\mathcal{C}_{0}\right)$ passe évidemment d'une représentation $\pi$ de $G$ à sa restriction $\pi_{\mid \Gamma}$, et de la Proposition 1.

Si $\pi=\operatorname{Ind}_{B}^{G}(\rho)$ est dans la série principale, on a de plus $\pi_{\mid \Gamma} \prec \lambda_{\Gamma}$. En effet, on a d'abord $\rho \prec \lambda_{B}$ car $B$ est moyennable, puis $\pi \prec \lambda_{G}=\operatorname{Ind}_{B}^{G}\left(\lambda_{B}\right)$ par continuité de l'induction, donc $\pi_{\mid \Gamma} \prec \lambda_{\Gamma}$ par le lemme.

Preuve du théorème 2. - L'assertion $\lambda_{\Gamma} \prec \pi_{t, \Gamma}$ est contenue dans le THÉORÈme 1. Par ailleurs $\pi_{t}$ n'est pas dans le support de la mesure de Plancherel sur $\widehat{G}$ [Lan, $\S$ VIII.4], donc $\pi_{t} \nprec \lambda_{G}\left[\mathrm{DC}^{*}, \mathrm{n}^{\circ}\right.$ 18.8.4]. Il résulte de l'assertion (ii) du lemme ci-dessus que $\pi_{t, \Gamma} \nprec \lambda_{\Gamma}$, ce qui montre (v).

Soient $s, t \in] 0,1\left[\right.$. Posons $\sigma=\operatorname{Ind}_{\Gamma}^{G}\left(1_{\Gamma}\right)$. Comme $\Gamma$ est un réseau dans $G$, on a $1_{G} \prec \sigma$ (c'est même vrai au sens fort). Pour toute représentation $\pi$ de $G$, on a donc :

$$
\pi \otimes 1_{G} \prec \pi \otimes \sigma=\operatorname{Ind}_{\Gamma}^{G}\left(\pi_{\mid \Gamma}\right) .
$$

Supposons que $\pi_{s, \Gamma} \prec \pi_{t, \Gamma}$. Alors on a :

$$
\pi_{s} \prec \operatorname{Ind}_{\Gamma}^{G}\left(\pi_{s, \Gamma}\right) \prec \operatorname{Ind}_{\Gamma}^{G}\left(\pi_{t, \Gamma}\right)=\pi_{t} \otimes \sigma .
$$

Notons $\widehat{G}_{c}$ l'adhérence dans $\widehat{G}$ pour la topologie de Fell de la série complémentaire, et décomposons $\sigma$ en $\sigma=\sigma_{1} \oplus \sigma_{2}$, où $\sigma_{1} \prec \lambda_{G}$ et $\sigma_{2} \prec \widehat{G}_{c}$. Comme

$$
\pi_{s} \nprec \lambda_{G} \quad \text { et } \quad \pi_{\mathrm{t}} \otimes \sigma_{1} \prec \pi_{\mathrm{t}} \otimes \lambda_{\mathrm{G}} \sim \lambda_{\mathrm{G}},
$$

il résulte de $\left(^{*}\right)$ qu'on a :

$$
\pi_{s} \prec \pi_{t} \otimes \sigma_{2} .
$$

Les produits tensoriels $\pi_{t} \otimes \pi_{t^{\prime}}$ ont été calculés par L. PUkAnszky [Rep], et on sait en particulier que :

- si $t+t^{\prime} \leq 1$, alors $\pi_{t} \otimes \pi_{t^{\prime}} \prec \lambda_{G}$;

- si $t+t^{\prime}>1$, alors il existe une représentation $\tau$ de $G$ telle que :

$$
\tau \prec \lambda_{G} \quad \text { et } \quad \pi_{t} \otimes \pi_{t^{\prime}}=\tau \oplus \pi_{t+t^{\prime}-1} .
$$

Il résulte donc de $\left(^{* *}\right)$ et de ce que $\pi_{t} \nprec \lambda_{G}$ qu'on a

$$
\pi_{s} \prec \bigoplus_{t^{\prime}: t^{\prime}>1-t} \pi_{t+t^{\prime}-1} \subset \bigoplus_{t^{\prime \prime}: t^{\prime \prime} \leq t} \pi_{t^{\prime \prime}}
$$

BULLETIN DE LA SOCIÉTÉ MATHÉMATIQUE DE FRANCE 
Ceci implique que $s \leq t$ en vertu de la bicontinuité de l'application $\tau \mapsto \pi_{\tau}$ (voir les articles [Del] et [EhM] déjà cités avant l'énoncé du THÉORÈME 3).

La preuve ci-dessus vaut pour tout sous-groupe discret $\Gamma$ de $G$ ayant les deux propriétés suivantes : $\Gamma$ est dense dans $G$ au sens de Zariski et l'espace $G / \Gamma$ est moyennable au sens d'Eymard. Comme exemple d'un tel groupe qui ne soit pas un réseau, on peut donner $\Gamma=\operatorname{Ker}\left(\Gamma_{g} \rightarrow \mathbb{Z}^{2 g}\right)$, où $\Gamma_{g}$ désigne un réseau dans $G$ isomorphe au groupe fondamental d'une surface close de genre $g \geq 2$, et où $\mathbb{Z}^{2 g}$ est identifié au premier groupe d'homologie de cette surface. (Ce groupe $\Gamma$ est bien Zariski-dense dans $G$, car la composante connexe de son adhérence de Zariski est un sous-groupe connexe de $G=\mathrm{PSL}_{2}(\mathbb{R})$ qui est non moyennable, donc égal à $G$ tout entier. Dans une autre terminologie, $\Gamma$ est un groupe fuchsien de première espèce qui n'est ni de type fini ni de covolume fini dans $G$.)

Preuve du théorème 3. - Par continuité de la restriction, l'application $t \mapsto \pi_{t, \Gamma}$ est continue.

Montrons que l'application $\pi_{t, \Gamma} \mapsto t$ est continue. Soit $A$ une partie de $] 0,1[$ et soit $s \in] 0,1$ [ tel que $\pi_{s, \Gamma}$ est dans l'adhérence de $\left\{\pi_{t, \Gamma}: t \in A\right\}$, c'est-à-dire tel que :

$$
\pi_{s, \Gamma} \prec \bigoplus_{t \in A} \pi_{t, \Gamma}
$$

Alors (voir la preuve du ThÉORÈme 2) :

$$
\pi_{s} \prec \bigoplus_{t \in A}\left(\pi_{t} \otimes \operatorname{Ind}_{\Gamma}^{G}\left(1_{\Gamma}\right)\right)
$$

Par ailleurs, la décomposition de $L^{2}\left(\mathrm{PSL}_{2}(\mathbb{Z}) \backslash \mathrm{PSL}_{2}(\mathbb{R})\right)$ sous l'action de $\mathrm{PSL}_{2}(\mathbb{R})$ est bien connue (voir [Ter, $\S 3.7$, th. 1, p. 254]). La série complémentaire n'apparaissant pas dans cette décomposition, on a

$$
\operatorname{Ind}_{\Gamma}^{G}\left(1_{\Gamma}\right) \prec 1_{G} \oplus \lambda_{G}
$$

et par suite :

$$
\pi_{s} \prec\left(\bigoplus_{t \in A} \pi_{t}\right) \oplus\left(\bigoplus_{t \in A}\left(\pi_{t} \otimes \lambda_{G}\right)\right) \prec\left(\bigoplus_{t \in A} \pi_{t}\right) \oplus \lambda_{G} .
$$

Comme $\pi_{s} \nprec \lambda_{G}$, on a donc :

$$
\pi_{s} \prec \bigoplus_{t \in A} \pi_{t}
$$

TOME $122-1994-\mathrm{N}^{\circ} 3$ 
La continuité de $\pi_{t} \mapsto t$ implique que $s \in \bar{A}$.

Il est clair que l'assertion (vii) du THÉORÈME 3 découle de la bicontinuité de $t \mapsto \pi_{t, \Gamma}$.

Nous remercions Alain VALETTE pour ses remarques après lecture d'une première version de ce travail.

\section{Ajouté sur épreuves.}

1) Comme nous l'a signalé G.A. WiLLIS, un groupe n'est jamais réunion d'un nombre fini de classes à gauche relatives à des sous-groupes dont les indices sont tous infinis. (C'est un résultat de B.H. NeumanN; voir J. London Math. Soc., t. 29, 1964, p. 236-248.) Il en résulte qu'un groupe cci est nécessairement fortement cci, au sens du numéro 3 ci-dessus.

2) Pour tout groupe $\Gamma$ comme au ThÉORÈme 1, nous savons maintenant que la $C^{*}$-algèbre réduite de $\Gamma$ est simple $[\mathrm{BCH}]$.

\section{BIBLIOGRAPHIE}

[BCH] BekKa (M.), Cowling (M.) and De la Harpe (P.). - Some Groups whose Reduced $C^{*}$-algebra is Simple, Prébublication, 1993.

[Bor] Borel (A.). - Density Properties for Certain Subgroups of Semisimple Lie Groups Without Compact Factors, Ann. of Math., t. 72, 1960, p. 179-188.

[CoS] Cowling (M.) and Steger (T.). - The Irreducibility of Restrictions of Unitary Representations to Lattices, J. Reine Angew. Math., t. 420, 1991 , p. 85-98.

[Del] Delorme (P.). - 1-cohomologie des représentations unitaires des groupes de Lie semi-simples et résolubles. Produits tensoriels continus de représentations, Bull. Soc. Math. France, t. 105, 1977, p. 281-336.

[DC*] Dixmier (J.). - Les $C^{*}$-algèbres et leurs représentations. - GauthierVillars, 1969 .

[EhM] Ehrenpreis (L.) and Mautner (F.I.). - Some Properties of the Fourier Transform on Semi-simple Lie Groups I, Ann. of Math., t. 61, 1955, p. 406-439.

[Eym] Eymard (P.). - Moyennes invariantes et représentations unitaires. - Springer, Lecture Notes in Math. 300, 1972. 
[Fel] Fell (J.M.G.). - Weak Containment and Induced Representations of Groups, Canad. J. Math., t. 14, 1962, p. 237-268.

[Gaa] GaAl (S.A.). - Linear Analysis and Representation Theory. Springer, 1973 .

[GHJ] Goodman (F.), de la Harpe (P.) and Jones (V.). - Coxeter Graphs and Towers of Algebras. - Springer, 1989.

[Har] DE la Harpe (P.). - Operator Algebras, Free Groups and Other Groups, Colloque d'Orléans, juillet 1992.

[HoM] Howe (R.E.) and Moore (C.C.). - Asymptotic Properties of Unitary Representations, J. Funct. Anal., t. 32, 1979, p. 72-96.

[HoR] Howe (R.E.) and Rosenberg (J.). - The Unitary Representation Theory of $\operatorname{GL}(n)$ of an Infinite Discrete Field, Israel J. Math., t. 67, 1989, p. 67-8. Voir aussi J. Rosenberg, C. R. Acad. Sc. Paris, t. 309, 1989 , p. $581-586$.

[Lan] LANG (S.). - $\mathrm{SL}_{2}(\mathbb{R})$. - Addison-Wesley, 1975.

[MvN] Murray (F.J.) and Von Neumann (J.). - On Rings of Operators, IV, Ann. of Math., t. 44, 1943, p. 716-808.

[Rep] RePka (J.).- - Tensor Products of Unitary Representations of $\mathrm{SL}_{2}(\mathbb{R})$, Amer. J. Math., t. 100, 1978, p. 747-774.

[Ter] Terras (A.). - Harmonic Analysis on Sammetric Spaces and Applications I. - Springer, 1985 .

[Wal] Wallach (N.R.). - Harmonic Analysis on Homogeneous Spaces. M. Dekker, 1973 .

[Zim] Zimmer (R.J.). - Ergodic Theory and Semisimple Groups. Birkhäuser, 1984 . 